\title{
JOINT NUMERICAL RANGES FOR UNBOUNDED NORMAL OPERATORS
}

\author{
by HUANG DANRUN
}

(Received 25th June 1984)

\section{Introduction}

For bounded operators, the theory of the joint numerical range has been developed by various authors $[1,2,3,4,5]$. Especially, the properties of commuting normal $n$-tuples are discussed in detail. Our purpose here is to show that many results in the above references still hold in the case of unbounded normal operators (see Theorem 2.3, Corrollary 3.5, Theorem 4.1, Theorem 4.2). Besides, the operator algebras are closely related to the theory of joint spectrum and joint numerical ranges in the bounded case (cf. $[1,3]$ ). How about unbounded operators? It seems that one must consider unbounded operator algebras. Some work has been done in this direction for the joint spectrum of unbounded normal operators [9]. In the last section of this paper, we provide some intimate relations between the joint numerical range and the unbounded operator algebras for unbounded normal operators.

Throughout this note, $H$ will be a complex Hilbert space with the scalar product ( , ). A closed operator $T$ on $H$ is said to be normal if $T$ is densely defined and $T^{*} T=T T^{*}$ (cf. [10]). We say an $n$-tuple of normal operators $A=\left(A_{1}, \ldots A_{n}\right)$ is commuting if their spectral measures are commutative. The joint spectrum $\operatorname{Sp}(A)$ of $A$ is defined as the support of $E=\prod_{i=1}^{n} E_{i}$, where $E_{i}$ is the spectral measure of $A_{i}, i=1, \ldots, n$.

The definitions here have their background in the multiparameter spectral theory (Sleeman [6]), and are closely connected with Taylor's joint spectrum of $A$ (see [9] and Section 5).

\section{Convexity of the joint numerical range}

Definition 2.1. Let $A=\left(A_{1}, \ldots, A_{n}\right)$ be a commuting $n$-tuple of normal operators, the joint numerical range of $A$ is the set $W(A)$ in $\mathbb{C}^{n}$ such that

$$
W(A)=\left\{\left(\left(A_{1} x, x\right), \ldots,\left(A_{n} x, x\right)\right): x \in D(A)=\bigcap_{i=1}^{n} D\left(A_{i}\right), \quad\|x\|=1\right\}
$$

where $D\left(A_{i}\right)$ is the domain of $A_{i}, i=1, \ldots, n$. 
We remark that $D(A)$ is a dense subspace of $H$ since it contains $E(\Delta) H$, where $E$ is the product spectral measure of $A$ and $\Delta$ is any compact subset of $\mathbb{C}^{n}$.

As far as we know, $W(A)$ is convex when $A$ is a commuting $n$-tuple of bounded normal operators or an $n$-tuple of Toeplitz operators [2,5]. In the following, we show that this is also true for a commuting $n$-tuple of unbounded normal operators.

Lemma 2.2. Suppose that $A=\left(A_{1}, \ldots, A_{n}\right)$ is a commuting $n$-tuple of normal operators. Then there exists a family $\left\{\left(\mathbb{C}^{n}, \rho_{\alpha}\right): \alpha \in \Lambda\right\}$ of measure spaces such that each $A_{k}$ is unitary equivalent to the maximal operator of multiplication by $z_{k}$ on $\oplus_{\alpha \in \Lambda} L_{2}\left(\mathbb{C}^{n} ; \rho_{\alpha}\right), k=1, \ldots, n$. That is

$$
D\left(A_{k}\right)=\left\{\left(f_{\alpha}\right) \in \underset{\alpha \in \Lambda}{\oplus} L_{2}\left(\mathbb{C}^{n} ; \rho_{\alpha}\right):\left(z_{k} f_{\alpha}\right) \in \underset{\alpha \in \Lambda}{\oplus} L_{2}\left(\mathbb{C}^{n}, \rho_{\alpha}\right)\right\}
$$

and

$$
A_{k}\left(f_{\alpha}\right)=\left(z_{k} f_{\alpha}\right), \text { for } \quad\left(f_{\alpha}\right) \in D\left(A_{k}\right), k=1, \ldots, n
$$

Proof. There exists the product spectral measure $E$ of $A$ since $A=\left(A_{1}, \ldots, A_{n}\right)$ is commuting and normal. It easily follows that

$$
A_{k}=\int_{\mathbb{C}^{n}} z_{k} d E, \quad k=1, \ldots, n
$$

The detailed proof is similar to that for single self-adjoint operators, we omit it here (cf. [10]).

Theorem 2.3. Let $A=\left(A_{1}, \ldots, A_{n}\right)$ be a commuting $n$-tuple of normal operators. Then the joint numerical range $W(A)$ is convex.

Proof. Without loss of generality, we may assume $A=\left(A_{1}, \ldots, A_{n}\right)$ to be the maximal operators of multiplication by $z=\left(z_{1}, \ldots, z_{n}\right)$ on $\bigoplus_{\alpha \in \Lambda} L_{2}\left(\mathbb{C}^{n} ; \rho_{\alpha}\right)$ by using Lemma 2.2.

Suppose that $f=\left(f_{\alpha}\right), g=\left(g_{\alpha}\right) \in D(A)=\bigcap_{i=1}^{n} D\left(A_{i}\right)$ and $\|f\|=\|g\|=1$, then for any $t \in[0,1]$ fixed, let $h=\left(h_{\alpha}\right)=\left(\left(t\left|f_{\alpha}\right|^{2}+(1-t)\left|g_{\alpha}\right|^{2}\right)^{1 / 2}\right)$. Since for any $k=1, \ldots, n$ and $\alpha \in \Lambda$,

$$
\sum_{\alpha} \int\left|z_{k} h_{\alpha}\right|^{2} d \rho_{\alpha}=\sum_{\alpha} t \int\left|z_{k} f_{\alpha}\right|^{2} d \rho_{\alpha}+\sum_{\alpha}(1-t) \int\left|z_{k} g_{\alpha}\right|^{2} d \rho_{\alpha}<+\infty
$$

we have $h=\left(h_{\alpha}\right) \in D(A)$. Furthermore, it is clear that

$$
\|h\|^{2}=\sum_{\alpha}\left\|h_{\alpha}\right\|^{2}=t \sum_{a}\left\|f_{\alpha}\right\|^{2}+(1-t) \sum_{\alpha}\left\|g_{\alpha}\right\|^{2}=1
$$


which implies

$$
\begin{aligned}
& t\left(\left(A_{1} f, f\right), \ldots,\left(A_{n} f, f\right)\right)+(1-t)\left(\left(A_{1} g, g\right), \ldots,\left(A_{n} g, g\right)\right) \\
= & \left(\sum_{\alpha} \int z_{1}\left(t\left|f_{\alpha}\right|^{2}+(1-t)\left|g_{\alpha}\right|^{2}\right) d \rho_{\alpha}, \ldots, \sum_{\alpha} \int z_{n}\left(t\left|f_{\alpha}\right|^{2}+(1-t)\left|g_{\alpha}\right|^{2}\right) d \rho_{\alpha}\right) \\
= & \left(\left(A_{1} h, h\right), \ldots,\left(A_{n} h, h\right)\right) \in W(A) .
\end{aligned}
$$

Hence $W(A)$ is a convex set in $\mathbb{C}^{n}$. This completes the proof.

\section{A characterization of the joint numerical range}

Definition 3.1. A point $z=\left(z_{1}, \ldots, z_{n}\right)$ of $\mathbb{C}^{n}$ is in the joint approximate point spectrum $\sigma_{\pi}(A)$ of $A$ if there exists a sequence $\left\{x_{i}\right\}$ of unit vectors in $D(A)=\bigcap_{i=1}^{n} D\left(A_{i}\right)$ such that

$$
\left\|\left(z_{k}-A_{k}\right) x_{i}\right\| \rightarrow 0 \quad(i \rightarrow \infty), k=1,2, \ldots, n .
$$

A point $z=\left(z_{1}, \ldots, z_{n}\right)$ of $\mathbb{C}^{n}$ is in the joint point spectrum $\sigma_{p}(A)$ of $A$ if there exists a nonzero vector $x$ in $D(A)$ such that

$$
A_{k} x=z_{k} x, \quad k=1, \ldots, n
$$

Lemma 3.2. Let $A=\left(A_{1}, \ldots, A_{n}\right)$ be a commuting $n$-tuple of normal operators. Then $\operatorname{Sp}(A)=\sigma_{\pi}(A)$ and $z_{0} \in \sigma_{p}(A)$ if and only if $E\left(\left\{z_{0}\right\}\right) \neq 0$ (cf. [9]).

Lemma 3.3. If $\Delta$ is a convex set in $\mathbb{C}^{n}$ and $\mu$ is a nonnegative Borel measure defined on $\Delta$ such that $\mu(\Delta)=1$, then

$$
\int_{\Delta} z d \mu(z) \in \Delta(\text { cf. [7]) }
$$

Now we proceed to prove the main result of this section.

Theorem 3.4. Let $E$ be the product spectral measure of a commuting $n$-tuple of normal operators $A=\left(A_{1}, \ldots, A_{n}\right)$, and let $S$ be the family of all the convex Borel sets $\Delta$ in $\mathbb{C}^{n}$ satisfying $E(S)=I$. Then

$$
W(A)=\bigcap_{\Delta \in S} \Delta
$$

Proof. We denote $V(A)=\bigcap_{\Delta \in S} \Delta$. 
(i) For any $\Delta \in S$ and $x \in D(A),\|x\|=1, \int_{\Delta} z d\|E(z) x\|^{2}$ is a Bochner integration about the probability measure $\mu_{x}=\|E(\cdot) x\|^{2}$. By Lemma 3.3 we have

$$
\begin{aligned}
\left(\left(A_{1} x, x\right), \ldots,\left(A_{n} x, x\right)\right) & =\left(\int_{\mathrm{sp}_{\left(A_{1}\right)}} z_{1} d\left(E_{1}\left(z_{1}\right) x, x\right), \ldots, \int_{\operatorname{Sp}_{\left(A_{n}\right)}} z_{n} d\left(E_{n}\left(z_{n}\right) x, x\right)\right) \\
& =\left(\int_{\Delta} z_{1} d(E(z) x, x), \ldots, \int_{\Delta} z_{n} d(E(z) x, x)\right) \\
& =\int_{\Delta} z d\|E(z) x\|^{2} \in \Delta .
\end{aligned}
$$

This proves that $W(A) \subset V(A)$.

(ii) Now we shall show that $V(A) \subset W(A)$.

It is known that $\operatorname{Sp}(A)=\sigma_{\pi}(A)$ (Lemma 3.2) and $W(A)$ is convex (Theorem 2.3), so we have $\operatorname{conv}(\operatorname{Sp}(A)) \subset \overline{W(A)}$. Suppose $z_{0} \in V(A)$, it is clear that $z_{0} \in \operatorname{conv}(\operatorname{Sp}(A))$.

If $E\left(\left\{z_{0}\right\}\right) \neq 0$, then $z_{0} \in \sigma_{p}(A) \subset W(A)$.

If $z_{0} \in \operatorname{int}(\operatorname{conv}(\operatorname{Sp}(A)))$, then $z_{0} \in \operatorname{int} \overline{W(A)} \subset W(A)$ since $W(A)$ is convex.

It remains to consider the case when $z_{0}$ is a boundary point of $\operatorname{conv}(\operatorname{Sp}(A))$ and $E\left(\left\{z_{0}\right\}\right)=0$.

Considering $\mathbb{C}^{n}$ as a real linear space of dimension $2 n$, we know that there exists a hyperplane $\pi$ of dimension $2 n-1$ which supports $\operatorname{conv}(\operatorname{Sp}(A))$ at $z_{0}$. Taking $z_{0}$ as origin, we shall establish a real rectangular coordinates system for $\mathbb{R}^{2 n}$ as follows.

First we can fix the $x_{1}$-axis such that the equation of $\pi$ is $x_{1}=0$ and $\operatorname{conv}(\operatorname{Sp}(A))$ is at the side of $x_{1} \geqq 0$.

In $x_{1}=0$, if for every open half-space $e$ one has $E(e) \neq 0$, then there must exist a compact convex set $\Delta \subset e$ such that $E(\Delta) \neq 0$. Hence we can pick a unit vector $x \in E(\Delta) H \subset D(A)$. By Lemma 3.3 we have

$$
\lambda_{e}=\left(\left(A_{1} x, x\right), \ldots,\left(A_{n} x, x\right)\right)=\int_{\Delta} z d\|E(z) x\|^{2} \in \Delta \subset e .
$$

Since $e$ is an arbitrary half-space in $\pi$, we can choose some points of kind $\lambda_{e}$ circles $z_{0}$. It follows from Theorem 2.3 that $z_{0} \in W(A)$.

On the other hand, if there exists a half-space $e$ in $\pi$ for which $E(e)=0$, then we fix the $x_{2}$-axis such that $e=\left\{x_{1}=0, x_{2}<0\right\}$. Replacing $\left\{x_{1}=0\right\}$ by $\left\{x_{1}=0, x_{2}=0\right\}$, we continue the procedure above. Then either for some step we obtain $z_{0} \in W(A)$ or eventually we get

$$
\begin{aligned}
& E\left(\left\{x_{1}<0\right\}\right)=0, \\
& E\left(\left\{x_{1}=0, x_{2}<0\right\}\right)=0, \\
& \cdots \cdots \cdots, \\
& E\left(\left\{x_{1}=x_{2}=\cdots=x_{2 n-2}=0, \quad x_{2 n-1}<0\right\}\right)=0 .
\end{aligned}
$$


Let $S_{1}=\left\{x_{1}=\cdots=x_{2 n-1}=0, x_{2 n}<0\right\}, S_{2}=\left\{x_{1}=\cdots=x_{2 n-1}=0, x_{2 n}>0\right\}$. We may assert that $E\left(S_{i}\right)=0, i=1,2$. Indeed, notice that $E\left(\left\{z_{0}\right\}\right)=0, E\left(S_{1}\right)=0$ would imply $E(M)=I$, where

$$
M=\left\{x_{1}>0\right\} \cup\left\{x_{1}=0, x_{2}>0\right\} \cup \cdots \cup\left\{x_{1}=\cdots=x_{2 n-1}=0, x_{2 n}>0\right\} .
$$

This is impossible since $M$ is convex and $z_{0}=(0, \ldots, 0) \notin M$. So we have proved $E\left(S_{1}\right) \neq 0$. Similarly, $E\left(S_{2}\right) \neq 0$. Then there must exist two compact convex sets $\Delta_{1} \subset S_{1}$ and $\Delta_{2} \subset S_{2}$ such that $E\left(\Delta_{i}\right)=0, i=1,2$. Thus we can pick unit vectors $x_{i} \in E\left(\Delta_{i}\right) H \subset D(A)$ and by Lemma 3.3 we have

$$
\eta_{i}=\left(\left(\mathrm{A}_{1} x_{i}, x_{i}\right), \ldots,\left(A_{n} x_{i}, x_{i}\right)\right) \in \Delta_{i} \subset S_{i}, \quad i=1,2 .
$$

Therefore, $z_{0} \in \operatorname{conv}\left\{\eta_{1}, \eta_{2}\right\} \subset W(A)$. The proof is complete.

Corollary 3.5. If $A=\left(A_{1}, \ldots, A_{n}\right)$ is a commuting $n$-tuple of normal operators, then

$$
\overline{W(A)}=\overline{\operatorname{conv}(\operatorname{Sp}(A))} \text {. }
$$

Proof. It immediately follows from the proof of Theorem 3.4.

\section{Boundary points of the joint numerical range}

By using Theorem 3.4, we can generalize some properties of the boundary points of $W(A)$ which were described in $[4,5]$ for the bounded case.

Theorem 4.1. Let $A=\left(A_{1}, \ldots, A_{n}\right)$ be a commuting $n$-tuple of normal operators. If $\lambda$ belongs to $\operatorname{Ext} \overline{W(A)}$ (the extreme points of $\overline{W(A)})$, then $\lambda \in \operatorname{Sp}(A)$.

Proof. Suppose $\lambda \in \operatorname{Ext} \overline{W(A)}$. If $\lambda$ does not belong to $\operatorname{Sp}(A)$, then there is a ball neighbourhood $0_{\lambda}$ of $\{\lambda\}$ such that $E\left(0_{\lambda}\right)=0$. One easily sees that $\left.M^{\prime}=\overline{\operatorname{conv}}(\overline{W(A)}) \backslash 0_{\lambda}\right)$ is a convex Borel set of $\mathbb{C}^{n}$ and $E\left(M^{\prime}\right)=I$. By Theorem 3.4 we have $\lambda \in \overline{W(A)} \subset \overline{M^{\prime}}=M^{\prime}$. But the facts $\lambda \in 0_{\lambda}$ and $\lambda \in \operatorname{Ext} \overline{W(A)}$ imply $\lambda$ does not belong to $M^{\prime}$. This is a contradiction.

Theorem 4.2. Let $A=\left(A_{1}, \ldots, A_{n}\right)$ be as above. If $\lambda \in W(A) \cap \operatorname{Ext} \overline{W(A)}$, then $\lambda \in \sigma_{p}(A)$.

Proof. Suppose $\lambda \in W(A) \cap \operatorname{Ext} \overline{W(A)}$. If $\lambda$ does not belong to $\sigma_{p}(A)$, then $E(\{\lambda\})=0$. It follows that $E(\overline{W(A)} \backslash\{\lambda\})=I$. We see that $\overline{W(A)} \backslash\{\lambda\}$ is still convex since $\lambda \in \operatorname{Ext} \overline{W(A)}$ and $\overline{W(A)}$ is convex. It follows from Theorem 3.4 that $\lambda \in \overline{W(A)} \backslash\{\lambda\}$. But this is a contradiction. This completes the proof. 


\section{Connections with unbounded operator algebras}

In this section, we shall consider some relations between the joint numerical range and the unbounded operator algebras for normal operators.

As before $A=\left(A_{1}, \ldots, A_{n}\right)$ is a commuting $n$-tuple of normal operators and $E$ the product spectral measure of $A$. Denote by $\mathscr{F}$ the family of all continuous functions on $\mathrm{Sp}(A)$. Consider the following set of operators:

$$
\mathscr{A}=\left\{E(f)=\int_{\mathrm{Sp}(A)} f d E: f \in \mathscr{F}\right\},
$$

We know already from [9] that $\mathscr{A}$ is a GB*-algebra as defined in G. Allan [8], whose topology is given by a collection of seminorms:

$$
T \rightarrow\left\|E\left(\Delta_{n}\right) T\right\|, \quad n=1,2, \ldots,
$$

where $T \in \mathscr{A}$ and $\left\{\Delta_{1} \subset \Delta_{2} \subset \cdots\right\}$ is a sequence of compact subsets in $\mathbb{C}^{n}$ which monotonically increase to $\mathbb{C}^{n}$. Let $\mathscr{D}=\bigcap_{f \in \mathscr{T}} \operatorname{Dom}(E(f))$, we also showed in [9] that $\mathscr{D}$ is a common core for $\mathscr{A}$ and $\mathscr{U}=\left.\mathscr{A}\right|_{\mathscr{D}}$ is an $\mathrm{EC}^{\#}$-algebra defined by A. Inoue [12]. We shall call $\mathscr{A}$ and $\mathscr{U}$ the $\mathrm{GB}^{*}$-algebra and the $\mathrm{EC}^{\#}$-algebra generated by $A=\left(A_{1}, \ldots, A_{n}\right)$ respectively.

In [9] we proved the following

$$
\begin{aligned}
\operatorname{Sp}_{E}(A)=\left\{\left(\chi\left(A_{1}\right), \ldots, \chi\left(A_{n}\right)\right):\right. & \chi \text { is the extended multiplicative } \\
& \text { linear functional on } \mathscr{A}\},
\end{aligned}
$$

and $\operatorname{Sp}(A)=\operatorname{Sp}_{E}(A) \cap \mathbb{C}^{n}$, where $\operatorname{Sp}_{E}(A)$. denotes the extended Taylor's joint spectrum of $A$.

Correspondingly, we have the following results referring to the joint numerical range of $A$.

First we recall that a functional $\phi$ on a $\mathrm{GB}^{*}$-algebra $\mathscr{A}$ is called a state if $\phi$ is positive in the sense $\phi\left(T^{*} T\right) \geqq 0$ for any $T \in \mathscr{A}$ and $\phi(I)=1$. Now we have

Theorem 5.1. Let $A=\left(A_{1}, \ldots, A_{n}\right)$ be a commuting $n$-tuple of normal operators and $\mathscr{A}$ the $G B^{*}$-algebra generated by $A$. Let

$$
U(A)=\left\{\left(\phi\left(A_{1}\right), \ldots, \phi\left(A_{n}\right)\right): \phi \text { is state on } \mathscr{A}\right\},
$$

then we have $\overline{W(A)}=\overline{U(A)}$.

Proof. Let $\mathscr{D}=\bigcap_{f \in \mathscr{F}} \operatorname{Dom}(E(f))$ as above, one easily sees from the proof of 3.4 that

$$
\begin{aligned}
W_{\mathscr{D}}(A) & \stackrel{\text { def }}{=}\left\{\left(\left(A_{1} x, x\right), \ldots,\left(A_{n} x, x\right)\right): x \in \mathscr{D},\|x\|=1\right\} \\
& =\bigcap_{\Delta \in S}=W(A) .
\end{aligned}
$$


Since for any $x \in \mathscr{D},\|x\|=1, T \rightarrow(T x, x)$ is a vector state on $\mathscr{A}$, we have $W(A)=$ $W_{\mathscr{A}}(A) \subset U(A)$.

Conversely, if there exists a state $\phi$ on $\mathscr{A}$ such that $\lambda_{0}=\phi(A) \notin \overline{W(A)}$, where $\phi(A)=$ $\left(\phi\left(A_{1}\right), \ldots, \phi\left(A_{n}\right)\right)$, then we can find a real hyperplane $\pi$ in $\mathbb{B}^{2 n}=\mathbb{C}$ which strictly separates $\lambda_{0}$ and $\overline{W(A)}$ since, from Theorem $2.3, \overline{W(A)}$ is a closed convex subset of $\mathbb{C}^{n}$.

Now let $\left\{\Delta_{m}\right\}$ be a sequence of compact sets in $\mathbb{C}^{n}$ which monotonically increase to $\mathbb{C}^{n}$ (see the first paragraph of this section). Obviously $A_{m}=E\left(\Delta_{m}\right) A=\left(E\left(\Delta_{m}\right) A_{1} \ldots, E\left(\Delta_{m}\right) A_{n}\right)$ is a commuting $n$-tuple of bounded normal operators and

$$
\operatorname{Sp}\left(A_{m}\right)=\left(\Delta_{m} \cap \operatorname{Sp}(A)\right) U\{(0, \ldots, 0)\} .
$$

There is no loss of generality in assuming that $(0, \ldots, 0) \in \mathrm{Sp}(A)$, since $\bar{W}(A), \bar{U}(A)$ and $\mathrm{Sp}(A)$ are all linear in $A$. Thus we have $\operatorname{Sp}\left(A_{m}\right) \subset \mathrm{Sp}(A), m=1,2, \ldots$ It follows by Corollary 3.5 that

$$
\overline{\bar{W}\left(A_{m}\right)}=\operatorname{conv}\left(\operatorname{Sp}\left(A_{m}\right)\right) \subset \overline{\operatorname{conv}(\operatorname{Sp}(A))}=\overline{\bar{W}(A)} .
$$

Let $C^{*}\left(A_{m}\right)$ be the unital $C^{*}$-subalgebra of $\mathscr{A}$ generated by $A_{m}$. Since Theorem 5.1 is known to be true for the bounded case [3] and $\phi$ is still a state when acting on $C^{*}\left(A_{m}\right)$, $m=1,2, \ldots$, we have $\phi$ is still a state when acting on $C^{*}\left(A_{m}\right), m=1,2, \ldots$, and $\phi\left(A_{m}\right) \in \overline{W\left(A_{m}\right)} \subset W(A)$. Besides, we can see that $\lim _{m \rightarrow \infty} E\left(\Delta_{m}\right) A_{i}=A_{i}, i=1, \ldots, n$, under the topology in $\mathscr{A}$ defined above. Since any positive linear functional on a complete symmetric unital algebra, whose topology is given by a countable family of seminorms, is continuous [13], we have $\phi\left(A_{m}\right) \rightarrow \phi(A)=\lambda_{0}$. But this is impossible because that $\lambda_{0}$ and $\overline{\bar{W}(A)}$ are separated strictly by the hyperplane $\pi$ in $\mathbb{C}^{n}$. Thus we have proved $U(A) \subset \overline{W(A)}$ and combining this with $W(A) \subset U(A)$ we get $\overline{U(A)}=\overline{W(A)}$. The proof is complete.

Remark. Theorem 5.1 generalizes the result in [3] since $U(A)$ is clearly a compact set when $A=\left(A_{1}, \ldots, A_{n}\right)$ are bounded.

Finally, we shall characterize $W(A)$ by means of the weakly continuous linear functional on $\mathscr{U}$ which is induced by $A$. Inoue [12].

Lemma 5.3. Let $\phi$ be a positive linear functional on the $E C^{\#}$-algebra $\mathscr{U}$ on $\mathscr{D}$. Then the following conditions are equivalent.

(1) $\phi$ is weakly continuous;

(2) $\phi(T)=\sum_{i=1}^{m}\left(T \xi_{i}, \xi_{i}\right)$ for some $\xi_{i} \in \mathscr{D}, i=1, \ldots, m$, and $T \in \mathscr{U}$.

This lemma comes from A. Inoue [12] and immediately leads to the following consequence.

Theorem 5.4. Let $A=\left(A_{1}, \ldots, A_{n}\right)$ be a commuting $n$-tuple of normal operators. $\mathscr{U}$ is the $E C^{\#}$-algebra generated by $A$. Let

$\mathscr{K}=\{\phi: \phi$ is weakly continuous positive functional on $\mathscr{U}, \phi(I)=1\}$. 
Then $W(A)=\left\{\left(\phi\left(A_{1}\right), \ldots, \phi\left(A_{n}\right)\right): \phi \in \mathscr{K}\right\} .\left(\right.$ Strictly, $\phi\left(A_{i}\right)$ should be written as $\left.\phi\left(A_{i} \mid \mathscr{D}\right).\right)$

Proof. By Lemma 4.3, any $\phi \in \mathscr{K}$ can be written in the form $\phi(T)=\sum_{i=1}^{m}\left(T \xi_{i}, \xi_{i}\right)$ for some $\xi_{i} \in \mathscr{D}, i=1, \ldots, m$, where $T \in \mathscr{U}$.

Since $\phi(I)=\sum_{i=1}^{m}\left\|\xi_{i}\right\|^{2}=1$, one easily sees that

$$
\begin{aligned}
& \left(\phi\left(A_{1}\right), \ldots, \phi\left(A_{n}\right)\right) \\
= & \sum_{i=1}^{m}\left\|\xi_{i}\right\|^{2}\left(\left(A_{1} \frac{\xi_{i}}{\left\|\xi_{i}\right\|}, \frac{\xi_{i}}{\left\|\xi_{i}\right\|}\right), \ldots,\left(A_{n} \frac{\xi_{i}}{\left\|\xi_{i}\right\|}, \frac{\xi_{i}}{\left\|\xi_{i}\right\|}\right)\right) \in W(A) .
\end{aligned}
$$

So we have $\left\{\left(\phi\left(A_{1}\right), \ldots, \phi\left(A_{n}\right)\right): \phi \in \mathscr{K}\right\} \subset W(A)$. The inverse inclusion is obvious. Thus the proof is complete.

The author would like to thank Professors Cheng Qixiang and Zhang Dianzhou, for their advice and encouragement during the preparation of this material. Thanks are also due to the referee for his or her valuable suggestions.

\section{REFERENCES}

1. F. F. Bonsall and J. Duncan, Numerical Ranges II (London Math. Soc. Lecture Notes Cambridge, 1973).

2. A. T. DASH, Joint numerical range, Glasnik Mat. 7 (1972), 75-81. 306.

3. J. J. Buoni and B. L. Wadhwa, On joint numerical ranges, Pacific J. Math. 77 (1978), 303-

4. P. JUNEJA, On extreme points of the joint numerical range of commuting normal operators, Pacific J. Math. 67 (1976), 473-476.

5. M. Cho and M. TAKAGUCHI, Boundary points of joint numerical ranges, Pacific J. Math. 95 (1981), 27-35.

6. B. D. Sleeman, Multiparameter Spectral Theory in Hilbert Space (London, Pitman, 1978).

7. H. Rubin and O. Wesler, A notation on convexity in Euclidian n-space, Proc. Amer. Math. Soc. 9 (1958), 522-523.

8. G. R. Allan, A spectral theory for locally convex algebra, Proc. London Math. Soc. 15 (1965), $399-421$.

9. Huang Danrun and Zhang Dianzhow, Joint spectrum and unbounded operator algebras, (to appear).

10. J. Weidmann, Linear Operators in Hilbert Space (Springer-Verlag, 1980).

11. E. Durszt, On the numerical range of normal operators, Acta Sci. Math. 25 (1964), 262-265.

12. A. Inoue, On a class of unbounded operator algebras, Pacific J. Math. 65 (1976), 77-95.

13. $\mathrm{X}_{\mathrm{IA}}$ DaoxING, Measures on Infinite Dimension Space and Integration Theory Vol. 1 (in Chinese) (Shanghai, 1965).

Department of Mathematics

East China Normal University

ShanghaI

ChIna 